\title{
Isolated mediastinal cystic lymphangioma in a child
}

Alpha Mathew Kavunkal, MCh, Jayavelan Ramkumar, MCh, Shivanand Gangahanumaiah, MCh, Kancheepuram N. Parimelazhagan, MCh, and Vijit Koshy Cherian, MCh, Tamil Nadu, India

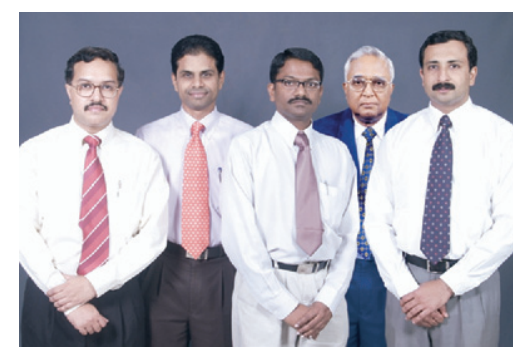

Drs Kavunkal, Ramkumar, Gangahanumaiah, Parimelazhagan and Cherian (left to right) ystic lymphangioma is a developmental malformation of the lymphatic system. As they develop in the body surface, $90 \%$ of all lymphangiomas are found by 2 years of age. ${ }^{1}$ However, those developing in the mediastinum, which account for less than $1 \%$, are mostly asymptomatic and are usually found accidentally in adulthood. ${ }^{2}$ We report here a rare case of isolated mediastinal cystic lymphangioma in a 3-year-old child.

\section{Clinical Summary}

A 3-year-old girl had had intermittent low-grade fever for 2 weeks. A chest radiogram showed a large homogeneous opacity in the left lower lung field (Figure 1). A computed tomographic scan revealed a well-defined $10 \times 5$-cm cystic lesion in the left paracardiac region in close contact with the heart (Figure 2). Superiorly, it extended up to the level of the superior pulmonary veins and, inferiorly, to the diaphragm with pressure effect on the left lower lobe and volume loss. The patient underwent left posterolateral thoracotomy. Intraoperatively, there was a large $10 \times 6$-cm cyst containing serous fluid in the anterior and middle mediastinum, which was adherent to the pericardium and diaphragm. The mediastinal cyst was excised and histopathologic examination revealed cystic lymphangioma. She had an uneventful postoperative period and is doing well 1 year after surgical excision.

\section{Discussion}

Cystic lymphangiomas, also known as hygromas, lymphatic cysts, or chylous cysts, are rare congenital abnormalities of the lymphatic vessels. These cysts are mostly present at birth, and the majority of them are detected before the age of 2 years. ${ }^{2,3}$ Although most of these cysts have been described in the pediatric population, adult patients with these cysts have also been described. Cystic lymphangiomas are most frequently located in the cervical $(75 \%)$ and axillary $(20 \%)$ regions. ${ }^{4}$ Isolated mediastinal cystic hygromas are very uncommon, being more common in the adult age group. In adults, these cysts are often recurrences of childhood tumors that were incompletely resected. Intrathoracic cysts are mostly located in the superior medias-

\footnotetext{
From the Department of Cardiothoracic Surgery, Christian Medical College \& Hospital, Vellore, Tamil Nadu, India.

Received for publication July 23, 2007; accepted for publication Aug 15, 2007.

Address for reprints: Alpha Mathew Kavunkal, MCh, Lecturer, Department of Cardiothoracic Surgery Unit 1, Christian Medical College \& Hospital, Vellore, 632 004, Tamil Nadu, India (E-mail: alphakavi@ hotmail.com).

J Thorac Cardiovasc Surg 2007;134:1596-7

$0022-5223 / \$ 32.00$

Copyright @ 2007 by The American Association for Thoracic Surgery doi:10.1016/j.jtcvs.2007.08.011
}

tinum. $^{4}$ Other cystic masses that must be considered in the differential diagnosis include teratoma, thymic cysts, necrotic tumors, pericardial or bronchogenic cysts, and goiters. The origin of lymphangiomas is controversial. They may be developmental, hamartomatous, or neoplastic.

These cysts in the mediastinum are mostly asymptomatic but may cause chest pain, cough, dyspnea, vocal cord paralysis, venous compression, or even stridor. Infection and hemorrhage commonly enlarge the cyst. The most common computed tomographic image is a well-circumscribed cystic mass in the anterior or superior mediastinum. Magnetic resonance imaging has the potential advantage of conclusive demonstration of cystic components, particularly in tumors with high attenuation on computed tomography because of high protein content and better demonstration of invasion of adjacent structures. Pathologically, these cysts were earlier subdivided into 3 groups: (1) capillary lymphangiomas composed of small lymphatics, (2) cavernous lymphangiomas composed of larger lymphatics, and (3) cystic lymphangiomas made up of large macroscopic lymphatic spaces with collagen and smooth muscle. This classification has largely been replaced by the all-inclusive term "lymphangioma" because the distinction among some of these lesions was arbitrary and, in fact, many lymphangiomas have both cystic and cavernous components. ${ }^{5}$

There are a few documented cases of spontaneous regression of cystic lymphangioma, but these occurred in sites other than the mediastinum. Surgical excision is the treatment of choice. Lymphangioma is a benign tumor, and a good prognosis is anticipated if the tumor is completely resected. However, it has been reported that the resected tissue contains infiltrative lesions and that recurrences are possible if the resection is incomplete.

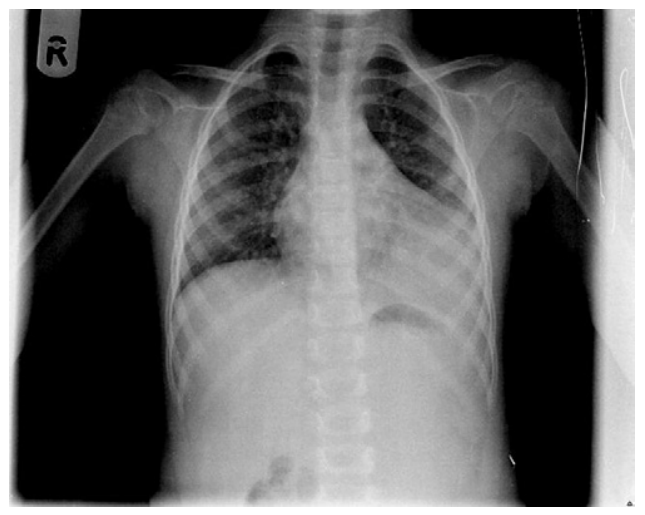

Figure 1. Chest radiogram shows a homogeneous opacity in the left lower lung field merging with the cardiac shadow. 


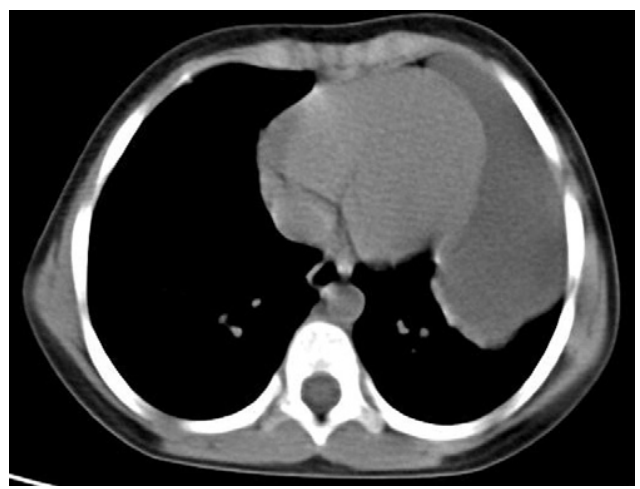

Figure 2. Computed tomographic scan of the chest revealing a large well-defined cyst in the left paracardiac region in close contact with pericardium and with pressure effect on the lower lobe.
Our patient had a large lymphangioma involving the anterior and middle mediastinum, which is unique inasmuch as isolated lymphangioma of the mediastinum in the pediatric population is exceedingly uncommon and there are only a few such reported cases. Complete resection of the cyst is the treatment of choice in these patients.

\section{References}

1. Feutz EP, Yune HY, Mandelbaum I, Brasher RE. Intrathoracic cystic hygroma: a report of three cases. Radiology. 1973;108:61-6.

2. Nansom EM. Lymphangioma (cystic hygroma) of the mediastinum. J Cardiovasc Surg (Torino). 1968;9:447-52.

3. Wright CC, Cohen DM, Vegunta RK, Davis T, King DR. Intrathoracic cystic hygroma: a report of three cases. J Pediatr Surg. 1996;31:1430-2.

4. Brown LR, Reiman HM, Rosenow EC 3rd, Gloviczki PM, Dirertie MB. Intrathoracic lymphangioma. Mayo Clin Proc. 1986;61:882-92.

5. Enzinger FM, Weiss SW. Soft tissue tumors. 4th ed. St Louis: Mosby; 2001. p. $955-83$

\section{Transcervical intraluminal repair of posterior membranous tracheal laceration through semi-lateral transverse tracheotomy}

In Kyu Park, MD, Jin Gu Lee, MD, Chang Young Lee, MD, Dae Joon Kim, MD, and Kyung Young Chung, MD, Seoul, Republic of Korea

\section{Clinical Summary}

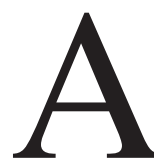

n 89-year-old female patient was admitted with pneumomediastinum in the left superior mediastinum on a chest radiogram after emergency orotracheal intuba-

tion in the emergency room. She was intubated owing to respiratory arrest after administration of sedatives to obtain a computed tomogram (CT) of the brain for evaluation of brain injury. She had arrived at the hospital with multiple injuries to the face and head in a stuporous mental status. The exact cause and vector of trauma were unknown. She had a history of dementia.

From the Department of Thoracic and Cardiovascular Surgery, Yonsei University College of Medicine, Seoul, Republic of Korea.

Received for publication June 13, 2007; revisions received Aug 1, 2007; accepted for publication Sept 5, 2007.

Address for reprints: In Kyu Park, MD, Department of Thoracic and Cardiovascular Surgery, Yonsei University College of Medicine, 250 Seongsanno, Seodaemun-gu, Seoul, Republic of Korea, 120-752 (E-mail: ik2653@yumc.yonsei.ac.kr).

J Thorac Cardiovasc Surg 2007;134:1597-8

$0022-5223 / \$ 32.00$

Copyright $\odot 2007$ by The American Association for Thoracic Surgery doi:10.1016/j.jtcvs.2007.09.001
Chest CT revealed tracheal rupture at the left posterolateral wall with subcutaneous emphysema, pneumomediastinum in the superior mediastinum, and pneumothorax on the left side. There was no abnormality on brain CT. On bronchoscopic scan, the trachea was lacerated longitudinally along the membranous and cartilage junction on the left. The distal end was $3 \mathrm{~cm}$ cephalad to the carina, but the proximal end was not defined. The balloon of the endotracheal tube was positioned distal to the laceration at the end of bronchoscopy.

After induction of general anesthesia, a cervical collar incision was made in the neck extension position. The left lateral wall of the trachea was dissected along the third and fourth tracheal cartilages. A tracheal laceration was found at the left posterior tracheal wall. The proximal end was at the level of fourth tracheal cartilage. The margin was sharp and clear. The distal end of the laceration was deep in the mediastinum because of the short, stiff neck. Transcervical extraluminal identification and repair of the laceration seemed impossible without a long segmental dissection of the trachea. To avoid extensive dissection and get appropriate access, we believed intraluminal repair through a tracheotomy to be the approach of choice.

A left semi-lateral transverse tracheotomy that connected to the laceration was made by a sharp blade to open the trachea in a reverse $\mathrm{L}$ shape. After retracting the corner of the reverse $\mathrm{L}$ with an anchoring suture, we could approach the whole laceration intraluminally (Figure 1). The laceration was about $4.5 \mathrm{~cm}$ long. It was repaired by interrupted $4-0$ absorbable monofilament sutures 